\title{
THE EFFECT OF PHASE ADVANCE ERRORS BETWEEN INTERACTION POINTS ON BEAM HALOS $\dagger^{\dagger}$
}

\author{
T. Chen, J. Irwin, R. H. Siemann, Stanford Linear Accelerator Center, Stanford University, Stanford, \\ CA 94309 USA
}

Phase advance errors between interaction points (IP) break the symmetry of multi-IP colliders. This symmetry breaking introduces new, lower order resonances which may change the halo from the beam-beam interaction dramatically. In this paper, the mechanism of introducing new resonances is discussed. Simulation results showing the changes due to phase advance errors are presented. Simulation results are compared with experimental measurements at VEPP-2M.

\section{INTRODUCTION}

The luminosity of circular $e^{+} e^{-}$colliders is usually limited by the lifetime caused by the beam-beam interaction. The mechanism that drives particles into the halo has been a puzzle, because factors combine in complex ways.

Understanding has been hampereded by the amount of CPU time required to simulate the halo. A method[1] was proposed to look into rare particles in the beam tail while saving a factor of hundreds, or even thousands, on CPU time. A program based on this method was written, tested and applied to PEP-II, LEP, CESR, and VEPP-2M to understand the halo from the beam-beam interaction. This study concluded that resonance streaming dominates the beam-beam lifetime[2].

The program has been upgraded to model multiple IP machines, such as LEP and VEPP-2M. The lattices between each individual IP are completely independent. Therefore, it can be used to investigate the effects of errors in each section. The results show the important role of the errors. Hamiltonian analysis has been extended to interpret the simulation results.

\section{HAMILTONIAN ANALYSIS FOR MULTIPLE IP'S WITH LATTICE ERRORS}

In existing multiple IP $e^{+} e^{-}$colliders, the IPs are symmetrically arranged so that, the collider can be treated as a few single IP colliders in cascade. However, when errors are introduced, especially when the phase advaces between IPs are different, this treatment is no longer valid. Because the differences are relatively small, we take them as the perturbations to the symmetric lattice.

The Hamiltonian including the beam-beam interaction can be written as

$$
H\left(x, p_{x}, y, p_{y}, s\right)=H_{0}+V_{B B}(x, y, s)
$$

where $H_{0}$ is the unperturbed Hamiltonian of the storage ring, and $V_{B B}$ is the beam-beam potential[3]. With $\mathrm{B}_{\mathbb{P}}$ interaction points, the beam-beam potential is

$$
\begin{gathered}
V_{B B}(x, y, s)=\frac{-N r_{e}}{\gamma} \sqrt{\frac{2}{\pi \sigma_{L}^{2}}} \sum_{n=-\infty}^{\infty} \sum_{b=0}^{B_{I P}-1} V_{F}(b, x, y, s) \\
\times \exp \left\{-\frac{2}{\sigma_{L}^{2}}\left[s-\left(n C+b \frac{C}{B_{I P}}+c \tau\right)\right]^{2}\right\} \\
\tau=\frac{\hat{\tau}}{2} \cos \left(2 \pi Q_{s}\left(n+\frac{b}{B_{I P}}\right)\right),
\end{gathered}
$$

and $V_{F}$ is defined in [3] with the additional feature that it depends on the parameters of the interaction point, $b$. By applying Fourier analysis, equation (1) becomes

$$
\begin{aligned}
& H=H_{0}-\frac{N r_{e}}{C \gamma} \sum_{b=0}^{B_{P p}-1} \sum_{m, n, p, r=-\infty}^{\infty} T_{p r}^{b} \exp \left\{2 \pi i\left(p \Delta Q_{x}^{b}+r \Delta Q_{y}^{b}\right)\right. \\
& \left.-\left(k_{p r}^{b} \sigma_{L}\right)^{2} / 8\right\} \times i^{m} J_{m}\left(k_{p r}^{b} \hat{\tau} c / 2\right) \exp \left\{i \left(p \psi_{x}+r \psi_{y}\right.\right. \\
& \left.\left.-2 \pi\left(n-m Q_{s}\right) s / C\right)+i 2 \pi n b / B_{I P}\right\}
\end{aligned}
$$

where $T_{p r}^{b}$ is a function of transverse actions and strongbeam size at each IP, and $k_{p r}^{b}$ is a wave number that also depends on IP parameters. The $\Delta Q^{b}$ s are the phase advance errors from one interaction point to the next one relative to the standard phase advance $Q / B_{I P}$.

Let's examine the phase in the second exponential function in equation (4). The last exponential function averages to zero, except when the resonance condition

$$
p Q_{x}+r Q_{y}+m Q_{s}=n,
$$

is satisfied. If there are no errors, i.e., all the IP's are identical and there are no phase advance differences, the superscript $b$ in eq. (4) can be dropped and the sum over $b$ is reduced to the factor $\sum_{b=0}^{B_{I P}-1} \exp \left\{i 2 \pi b n / B_{I P}\right\}$. This factor, which can be viewed as a sum of unit-length phasors, equals zero unless $n$ is a multiple of $B_{I P}$. Resonances with $n$ not equal to a multiple of $B_{I P}$ are eliminated because the phasors cancel each other. The resonances left are

$$
p \frac{Q_{x}}{B_{I P}}+r \frac{Q_{y}}{B_{I P}}+m \frac{Q_{s}}{B_{I P}}=\text { integer. }
$$

This is equivalent to a storage ring with one IP and $1 / B_{I P}$ of the size. If the IP's are not identical, or there are phase advance errors, or both, the cancellation does not 
occur. When the IP's are not identical, the phasors have unequal magnitudes, so that they will not cancel completely. When the phase advances between IP's are different, the phasors are no longer evenly spaced, and the cancellation is incomplete.

The above analysis gives two consequences for multiple IP colliders with errors: First, more resonances are introduced. The resonance condition with errors is $p Q_{x}+r Q_{y}+m Q_{s}=n$, or, in terms of tune per IP, the condition is

$$
p \frac{Q_{x}}{B_{I P}}+r \frac{Q_{y}}{B_{I P}}+m \frac{Q_{s}}{B_{I P}}=\frac{\text { integer }}{B_{I P}} .
$$

Comparing eq. (7) with eq. (6), one can see that many more resonances are allowed in this case. Second, among those resonances, some are possibly of lower order. They can dramatically change the tail distribution and the lifetime.

\section{SIMULATION OF A 4-IP MACHINE-LEP}

The first multi-IP machine are studied was LEP, which has 4 interaction points symmetrically distributed. Large synchrotron radiation energy losses and chromatic effects naturally break the symmetry. The tune errors can be as large as 0.015 to 0.04 because only two arcs have RF cavities[4]. The errors introduced in the simulation include errors in the $\beta$-functions and dispersion functions at each IP, and phase advance errors between IP's. When phase advance errors are included, the total tunes of the storage ring are held constant.

The beam distribution from simulations is plotted in transverse amplitude space. The amplitudes are normalized to beam sizes. The contour lines indicate equal number density and are spaced logarithmically. Figure 1 gives the beam distribution with a linear lattice and 4 symmetric IP's. Resonance lines allowed by symmetry up to 8th order are plotted over the distribution. One can see that the sixth order resonance $2 Q_{x}-2 Q_{y}-2 Q_{s}=1$ dominates the tail formation.

When lattice errors are included, the tail distribution changes dramatically, as shown in figure 2. About 40 resonances appeared inside the footprint of the beam-beam interaction. We have plotted four of those that appear related to the halo distribution. The 6 resonances in figure 1 are still present, but they now appear to have little effect on the tail distribution. The resonances $2 Q_{x}+2 Q_{y}+0 Q_{s}=5 / 4$ and $4 Q_{x}+0 Q_{y}-3 Q_{s}=9 / 4$ seem responsible for the vertical tail. The resonance $2 Q_{x}+2 Q_{y}-1 Q_{s}=5 / 4$ also has an effect at the upper left corner. Notice that all these resonances are forbidden in the symmetrical case.

The horizontal tail is believed to be related to the resonance $4 Q_{x}=9 / 4$, another low order resonance forbidden by symmetry. As a result, a peak at $A_{x}=5.5, A_{y}=1$ is formed. The horizontal tune of LEP is 0.2756 , which is close to the 4th integer resonance, making this resonance strong. Figure 3 plots the lifetime as a function of horizontal aperture for the symmetric lattice and the lattice with errors. The $4 Q_{x}=9 / 4$ would cause a short lifetime if the horizontal aperture was below $A_{x} \sim 9$.

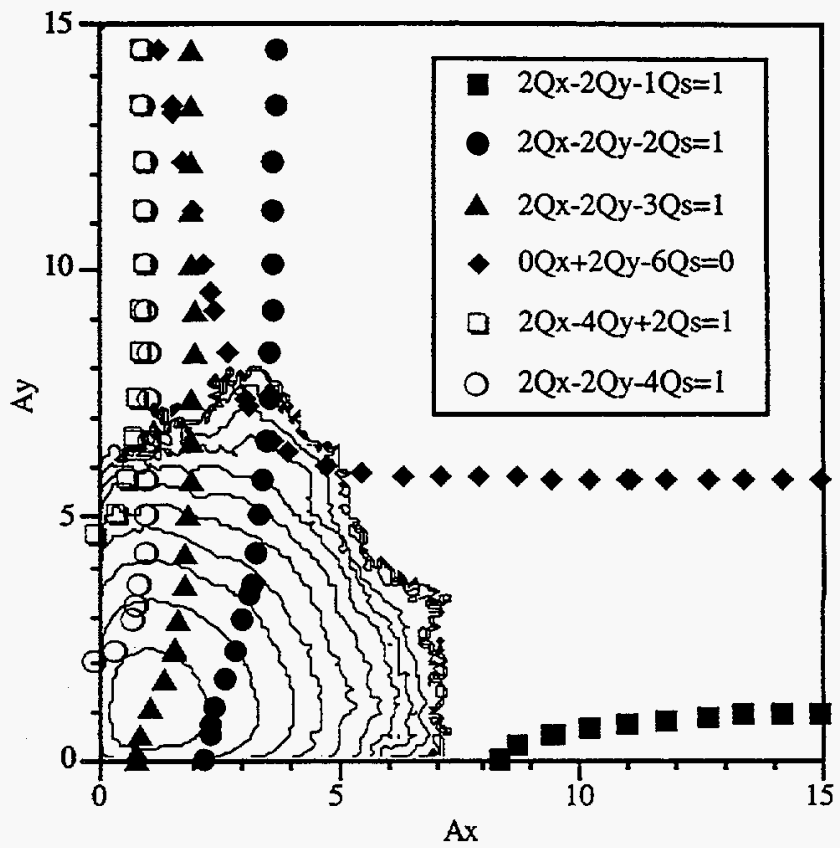

Figure 1. LEP beam-beam tail distribution and resonances. A linear, symmetric lattice is used. The tunes in the legend refer to $1 / 4$ of the total ring tunes.

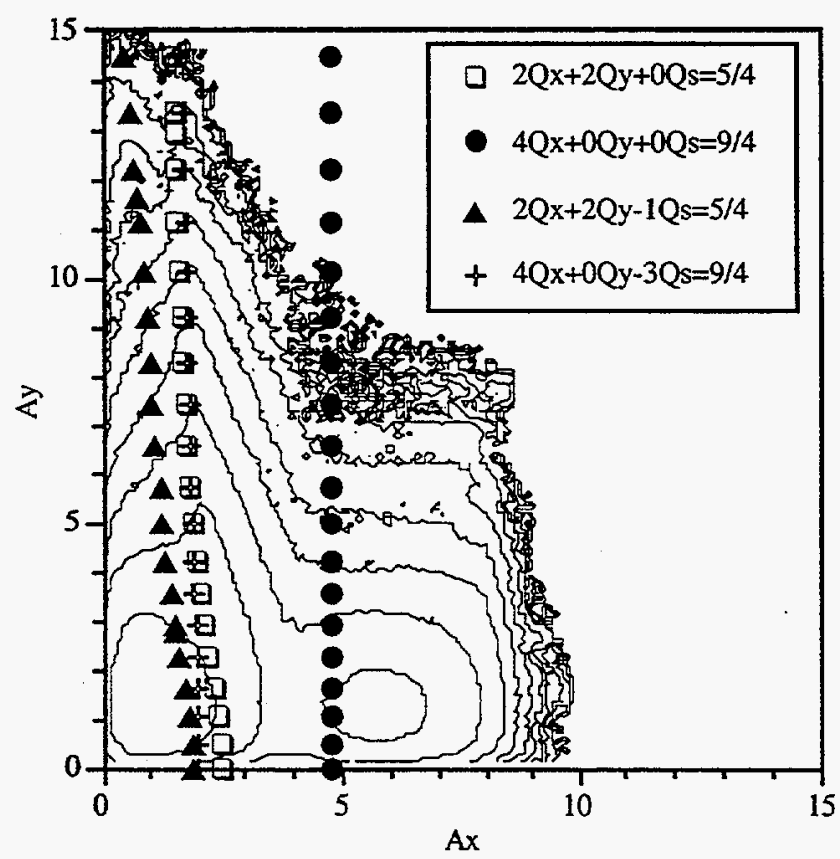

Figure 2. LEP beam-beam tail distribution when lattice errors are included. The tunes in the legend refer to $1 / 4$ of the total ring tunes. 


\section{DISCLAIMER}

This report was prepared as an account of work sponsored by an agency of the United States Government. Neither the United States Government nor any agency thereof, nor any of their employees, makes any warranty, express or implied, or assumes any legal liability or responsibility for the accuracy, completeness, or usefulness of any information, apparatus, product, or process disclosed, or represents that its use would not infringe privately owned rights. Reference herein to any specific commercial product, process, or service by trade name, trademark, manufacturer, or otherwise does not necessarily constitute or imply its endorsement, recommendation, or favoring by the United States Government or any agency thereof. The views and opinions of authors expressed herein do not necessarily state or reflect those of the United States Government or any agency thereof. 


\section{DISCLAIMER}

Portions of this document may be illegible in electronic image products. Images are produced from the best available original document. 


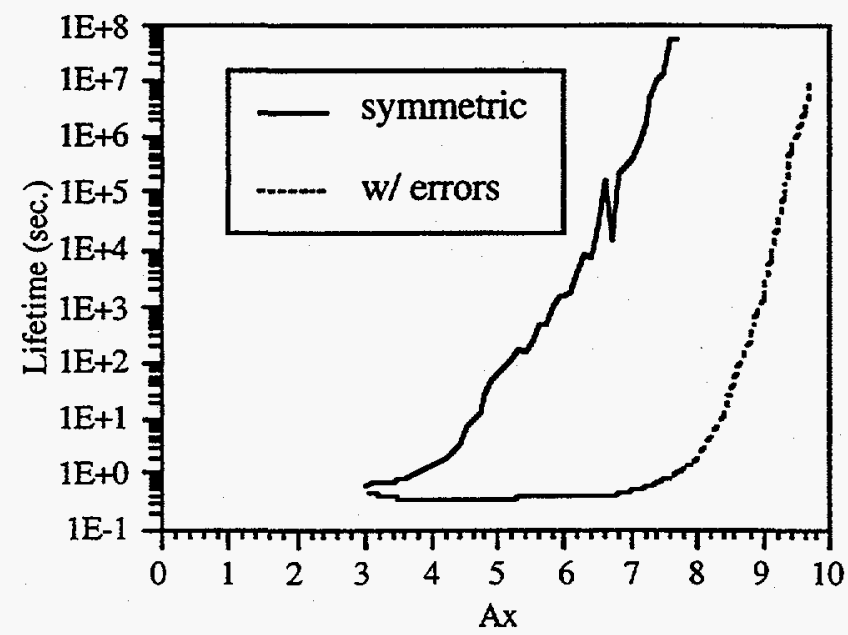

Figure 3. Lifetime versus horizontal aperture for LEP with and without errors.

\section{SIMULATION RESULTS COMPARED WITH VEPP-2M MEASUREMENT}

The effects of phase advance errors have been measured in VEPP-2M[5] which has a two-fold symmetry. This offers the opportunity to compare our simulation with data. Parameters are given in Table $1[5,6]$. The maximum beam-beam tune-shift was determined by the lifetime falling to about $500 \mathrm{sec}$, and it was measured as a function of phase advance error.

The first step of the simulation was to set the phase advance error to zero and set the beam current to give a beam-beam tune-shift of 0.045 as measured. The lifetime versus vertical aperture was calculated under these conditions, and the vertical aperture for a 500 second lifetime was determined. It was $A_{y}=29$. Then phase advance errors were introduced and the current adjusted until the lifetime was 500 second with $A_{y}=29$. The beambeam tune-shift at this current is plotted in figure 4 .

Table 1. VEPP-2M parameters

\begin{tabular}{|c|c|l|c|}
\hline \hline $\mathrm{Q}_{x}$ & 0.059 & $\mathrm{E}_{0}(\mathrm{MeV})$ & 510 \\
\hline $\mathrm{Q}_{\mathrm{y}}$ & 0.097 & $\mathrm{U}_{0}(\mathrm{KeV})$ & 9.17 \\
\hline $\mathrm{Q}_{s}$ & 0.0085 & $\varepsilon_{\mathrm{x}}(\mathrm{m} \bullet \mathrm{rad})$ & $4.6 \times 10^{-7}$ \\
\hline$\beta^{*}{ }_{\mathrm{x}}(\mathrm{m})$ & 0.48 & $\varepsilon_{\mathrm{y}}(\mathrm{m} \bullet \mathrm{rad})$ & $4.1 \times 10^{-9}$ \\
\hline$\beta^{*}{ }_{\mathrm{y}}(\mathrm{m})$ & 0.048 & $\mathrm{~T}_{\mathrm{n}}(\mathrm{sec})$ & $6 \times 10^{-8}$ \\
\hline$\eta^{*}{ }_{\mathrm{x}}(\mathrm{m})$ & 0.4 & $\alpha$ & 0.33 \\
\hline
\end{tabular}

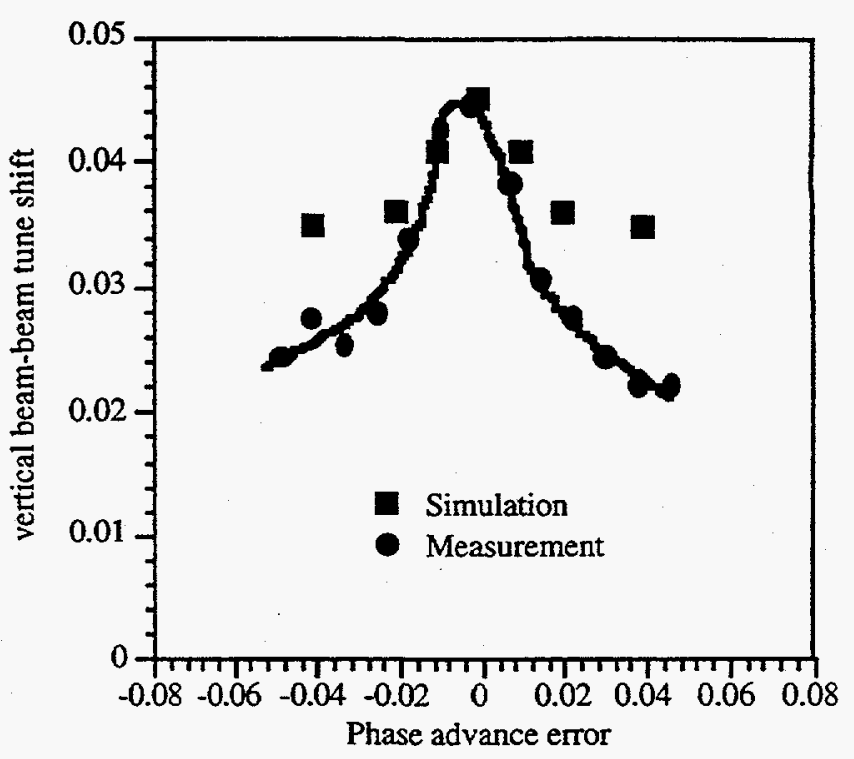

Figure 4 The maximum beam-beam tune-shift as a function of phase advance errors, simulation and measurement[5].

The simulation and the measurement show that the best performance is obtained with no errors. The quantitative agreement is not good, with the simulation giving more optimistic results than the experiment. This may be due to lattice nonlinearities which are not included in the present simulation.

\section{REFERENCES}

[1] J. Irwin, Proc of the 3rd Advanced ICFA Beam Dvnamics Workshop. Novosibirsk, USSR, 123 (1989) ISLAC-PUB-5743,Feb. 1992.

[2] T. Chen, J. Irwin and R. Siemann, Physical Review E, Vol. 49, No. 3, p2323, March 1994.

[3] R. Siemann, in Frontiers of Particle Beams with $e^{+} e^{-}$ Rings edited by M. Dienes, M. Month, B. Strasser and S. Turner(Springer-Verlag, Berlin, 1994),p. 327.

[4] J. P. Koutchouk, Proc. of the 1987 Particle Accelerator Conference. Washington D.C., 1987/CERN LEPTH/87-16.

[5] P. M. Ivanov, et al, Proc. of the 3rd Advanced ICFA Beam Dynamics Workshop. Novosibirsk, USSR, 26 (1989) /SLAC-PUB-5743,Feb. 1992.

[6] P. M. Ivanov, private communication. 